\title{
MEASURING BEHAVIOR 2002 Editorial
}

The articles in the August 2003 issue of Behavior Research Methods, Instruments, \& Computers are based on presentations that were made at the 4th International Conference of Methods and Techniques in Behavioral Research, held at the Vrije Universiteit in Amsterdam, The Netherlands, on August 27-30, 2002. The program chair and conference host was Dr. Gerrit C. van der Veer, Department of Computer Science, Vrije Universiteit, Amsterdam, The Netherlands.

Measuring Behavior is a periodic conference on methods and techniques in behavioral research, which serves to create bridges between disciplines by bringing together people with common interests. At a Measuring Behavior meeting, one finds ethologists; behavioral ecologists; behavioral neuroscientists; developmental, social, and work psychologists; psychiatrists; linguists; psychophysiologists; toxicologists; ergonomists, human factors researchers, movement scientists, and usability testers. Despite the diversity of research questions and applications across fields, all delegates share an interest in methods, techniques, and tools for the study of human or animal behavior.

Measuring Behavior 2002 brought together more than 325 delegates from 37 countries. During 4 conference days, there were 150 presentations grouped in 10 thematic symposia. Delegates read papers, presented posters, and demonstrated software or equipment. The program also included scientific tours, technical tutorials, special interest groups, training sessions, and user meetings organized by vendors, as well as an exhibition of scientific books, instruments, and software.

The articles published in this issue of BRMIC illustrate the variety of technical topics addressed at a Measuring Behavior meeting. Of course they represent only a small selection of the presentations made at the conference. The proceedings may be found at http://www.noldus.com/events/mb2002/ index.html. Printed copies of the proceedings can be obtained from the organizers, Noldus Information Technology, P. O. Box 268, 6700 AG Wageningen, The Netherlands (fax: +31-317-424496; email:mb2002@ noldus.nl).

Sixteen research groups have provided articles for the Measuring Behavior issue of BRMIC. Carletta et al. describe new tools for observing multimodal behavior. Papers by Baravalle and Lanfranchi; Paganelli and Paternò; and Faulkner address human-computer interaction, the developing field of usability testing. Hoogeboom, as well as Jansen, Wiertz, and Meyer, addresses the challenges of acquiring reliable and valid observational data from multiple sources or multiple observers. Gazit and Chen observe how participants represent a virtual solar system, and de Ruiter et al. investigate how information is used in dyadic interactions.

The papers by Cohn et al., Veeger et al., and Kastberger and Stachl explore how to record such indicators as eye blinks, shoulder movements, and aspects of behavior that may be observable only in the infrared spectrum.

Finally, papers by Melnick and Dow-Edwards, Berger et al., Reise et al., and Tilborg et al. address the technical aspects of studying such diverse behavioral systems as tobacco hornworm feeding, murine brain development after drug exposure, human cardiac sympathetic activity, and the odorantdependent locomotion of mites.

Information about forthcoming Measuring Behavior conferences can be found at http:// www.noldus.com/events/.

Jonathan Vaughan

Hamilton College 